\title{
NONLOCAL SECOND-ORDER GEOMETRIC EQUATIONS ARISING IN TOMOGRAPHIC RECONSTRUCTION
}

\author{
ALI SROUR
}

\begin{abstract}
In this paper, we study a new model of nonlocal geometric equations which appears in tomographic reconstruction when using the level-set method. We treat two additional difficulties which make the work original. On one hand, the level lines do not evolve along normal directions, and the nonlocal term is not of "convolution type". On the other hand, the speed is not necessarily bounded compared to the nonlocal term. We prove a existence and uniqueness results of our model.
\end{abstract}

In this paper, we study a fully nonlinear parabolic equation with nonlocal term. More precisely,

$$
\left\{\begin{array}{l}
\frac{\partial u}{\partial t}(x, t)=F\left(x, t, D u, D^{2} u, K_{x, t, u}^{k,+}\right) \quad \text { in } \mathbb{R}^{\mathrm{N}} \times[0, \mathrm{~T}], \\
u(x, 0)=u_{0}(x) \quad \text { in } \mathbb{R}^{N} \times\{0\},
\end{array}\right.
$$

where $N \geq 1$ is an integer, $T>0$. The unknown function is $u: \mathbb{R}^{N} \times$ $[0, T] \longrightarrow \mathbb{R}, D u$ and $D^{2} u$ denote respectively the gradient and the Hessian of $u$ with respect to the space variable, $u_{0}: \mathbb{R}^{N} \longrightarrow \mathbb{R}$ is the given initial data and $K_{x, t, u}^{k,+}$ denotes the nonlocal term, given for some integer $k \leq N$ by

$$
K_{x, t, u}^{k,+}=[u]_{x, t}^{+} \cap A_{x}^{k}
$$

where

and

$$
[u]_{x, t}^{+}=\left\{y \in \mathbb{R}^{N}: u(y, t) \geq u(x, t)\right\}
$$

$$
A_{x}^{k}=\left\{y=\left(y_{1}, \ldots, y_{k}, y_{k+1}, \ldots, y_{N}\right) \in \mathbb{R}^{N}: \pi_{k}(y)=\pi_{k}(x)\right\}
$$

where $\pi_{k}$ denote the projection function from $\mathbb{R}^{N}$ into $\mathbb{R}^{k}$ defined by

$$
\pi_{k}(x)=\left(x_{1}, \ldots, x_{k}\right)
$$

for all $x=\left(x_{1}, \ldots, x_{k}, x_{k+1}, \ldots, x_{N}\right)$. In the same manner

$$
K_{x, t, u}^{k,-}=[u]_{x, t}^{-} \cap A_{x}^{k}
$$

where

$$
[u]_{x, t}^{-}=\left\{y \in \mathbb{R}^{N}: u(y, t)>u(x, t)\right\} .
$$

Date: October 12, 2018.

Key words and phrases. Nonlocal Hamilton-Jacobi equations, Tomographic reconstruction, Nonlocal front propagation, level-set approach, Viscosity solutions. 
The nonlinearity $F$ is a continuous function from $\mathbb{R}^{N} \times \mathbb{R} \times \mathbb{R}^{N} \backslash\{0\} \times \mathscr{S}_{N} \times$ $\mathscr{B}_{N-k}$ into $\mathbb{R}$, where $\mathscr{S}_{N}$ is the set of real symmetric $N \times N$ matrices and $\mathscr{B}_{N-k}$ is the set of equivalence classes of all subsets of $\mathbb{R}^{N-k}$ with respect the relation $A \sim B$ if $\mathcal{L}^{N-k}(A \Delta B)=0$, where $\mathcal{L}^{N-k}$ is the Lebesgue measure on $\mathbb{R}^{N-k}$.

We consider $\mathscr{B}_{N-k}$ with a topology that comes from the metric

$$
d(A, B)=\sum_{n=0}^{\infty} \frac{\mathcal{L}^{N-k}((A \Delta B) \cap B(0, n))}{2^{n} \mathcal{L}^{N-k}(B(0, n))}
$$

where $B(0, n)$ denotes the ball in $\mathbb{R}^{N-k}$ of center 0 and radius $n$. With this topology a sequence $\left(K_{n}^{k}\right)_{n \geq 1}$ in $\mathscr{B}_{N-k}$ converges to $K^{k} \in \mathscr{B}_{N-k}$ if and only if $\mathbb{1}_{K_{n}^{k}}$ converges to $\mathbb{1}_{K^{k}}$ in $L_{\text {loc }}^{1}\left(\mathbb{R}^{N-k}\right)$.

We are interested in equations of type (11) which are related with tomographic reconstruction using active curves and the level-set approach [12, 7]. The model case we have in mind is

$$
F\left(x, t, p, X, K_{x, t, u}^{1,+}\right)=C(x, t)\left(\int_{K_{x, t, u}^{1,+}} g(z) d z\right)|p|+\operatorname{Trace}\left[\left(I-\frac{p \otimes p}{|p|^{2}}\right) X\right]
$$

where $x=\left(x_{1}, x_{2}\right), K_{x, t, u}^{1,+}=[u]_{x, t}^{+} \cap A_{x}^{1}, g$ is a positive function and $C$ is positive and Lipschitz continuous function.

We recall that the level-set approach was first introduced by Osher and Sethian [13] for numerical computations, and then developed from a theoretical point view by Evans and Spruck [10] for motion by mean curvature and by Chen, Giga and Goto 8 for general normal velocities. We also refer the reader to Barles, Soner and Souganidis [3] and Souganidis [15, 16] for different presentations and other results on the level-set approach.

In [14 Slepčev studied the motion of fronts in bounded domains by normal velocities which can depend on non local terms, in addition to the curvature, the normal direction and the location of the front. In fact, the velocities depend on non local terms, if the velocities at any point of the front depend on the set that the front encloses. Depending on the velocities, the motion of the front can be described by the partial differential equation

$$
\frac{\partial u}{\partial t}(x, t)=F\left(x, t, D u(x, t), D^{2} u(x, t),[u]_{x, t}^{+}\right) \quad \text { in } \quad O \times[0, T]
$$

with the Neumann boundary conditions $\partial u / \partial \gamma=0$ on $\partial O \times[0, T]$ and $u(x, 0)=u_{0}(x)$, where $O$ is a bounded domain in $\mathbb{R}^{\mathbb{N}}$. Slepčev proved an existence and uniqueness result for this equation, using the viscosity solution. 
In [2], Barles, Cardaliaguet, Ley, Monneau studied the first-order nonlocal equation

$$
\left\{\begin{array}{l}
\frac{\partial u}{\partial t}(x, t)=\left(C_{0}(., t) * \mathbb{1}_{[u]_{x, t}^{+}}(x, t)+C_{1}(x, t)\right)|D u| \quad \text { in } \mathbb{R}^{N} \times[0, T], \\
u(x, 0)=u_{0}(x) \quad \text { in } \mathbb{R}^{N} \times\{0\} .
\end{array}\right.
$$

This equation appears when modelling dislocations in crystals using the level set approach. The $*$ denotes the convolution in space, $C_{0}$ and $C_{1}$ are two functions on which we have some conditions. They proved an existence and uniqueness result for this equation in both cases where $C_{0}$ is a positive and a negative function, $C_{0}(., t) \in L^{1}\left(\mathbb{R}^{N}\right)$ for all $t \in[0, T]$ and $C_{0}, C_{1}$ are two Lipschitz continuous functions .

Now a question comes out: what are the differences between our equation (11) and the equations (3) and (4)? To explain the differences between our equation (11) and the equation (3) for example, we consider the case $N=2$ and the typical equation (2).

In (4), for any $(x, t) \in \mathbb{R}^{2} \times[0, T]$, the nonlocal term is given by

$$
[u]_{x, t}^{+}=\left\{y \in \mathbb{R}^{2}: u(y, t) \geq u(x, t)\right\} .
$$

and we integrate on a subset of $\mathbb{R}^{2}$. In our Equation (2), the new nonlocal term is given for any $(x, t) \in \mathbb{R}^{2} \times[0, T]$ by

$$
K_{x, t}^{1,+}=[u]_{x, t}^{+} \cap A_{x}^{1}=\left\{y \in \mathbb{R}^{2}: u(y, t) \geq u(x, t)\right\} \cap\left\{y_{1}=x_{1}\right\}
$$

and we integrate in (2) over a straight line i.e. the second variable is always fixed. We remark that the technique used in 2, 14 cannot be applied in the case of this new nonlocal term. We change the dependence in $D u$, we are able to prove a uniqueness and existence result of our equation (11).

Moreover, contrary to the cases studied in 2, 14, in the initial compact front, we allows a not bounded dependence of volume in our equation (11) (see (H5-1) and (H6-1)).

Let us now explain how this paper is organized: in Section 2, we present our model and we recall the definition of viscosity solutions. In Section 3, we prove a uniqueness result for our model with compact fronts and in Section 4 we show a uniqueness result for non compact fronts. In Section 5 , we prove an existence result for compact and non compact fronts. Finally, we give, in Appendix, the proof of the stability result for our model.

\section{Presentation of our model}

Tomography processes are widely studied by many authors. Most of cases concern the images restoration from a lot of projected data. In [17, M. Somekh addresses the problem of reconstruction of an image from two pairs of its orthogonal projections. The paper by Dinten, Bruandet and Peyrin [7] addresses tomographic reconstruction of binary objects from a small number of noisy projections in applications where the global dose remains constant 
with an increase or a decrease of the number of projections. Here we are interested in the reconstruction method of [12, where the authors consider a single view of tomographic reconstruction for radially symmetric objects and binary image. They formulate the problem as a front propagation which consists in evolving the contour of the noisy image (initial front), with a selected normal velocity, so that it converges toward contour of the initial object. This evolution is described by an approach of level-set which leads a nonlocal equation

$$
\left\{\begin{array}{l}
\frac{\partial u}{\partial t}=C(x, t)\left(\int_{\mathbb{R}} \mathbb{1}_{\left\{u\left(., x_{2}, t\right) \geq 0\right\}}(z) g(z) d z\right)|D u|+\text { Trace }\left[\left(I-\frac{D u \otimes D u}{|D u|^{2}}\right) D^{2} u\right], \\
u(x, 0)=u_{0}(x) \text { in } \mathbb{R}^{N} \times\{0\}
\end{array}\right.
$$

where $x \in \mathbb{R}^{2}, g$ is a positive functions and $g \in L^{1}(\mathbb{R})$. The second term on the right hand side of (5) is the mean curvature. This term was studied in [10] where it was used to regularize the evolution of the initial front with normal velocity. This equation (5) has been studied in [12] with $C \leq 0$, but in this papers we change the sign of $C$ in order to keep the assumption of monotony (see (H3)) which is a classical assumption to provide comparison and uniqueness results. From this equation, since $x_{2}$ is fixed, a discontinuity appears in the normal speed of propagation. To see this problem of continuity it is enough to start with a rectangular initial front whose sides are parallel to the coordinate axes and this rectangle can be represented as a level-set zero of a function $u$ from $\mathbb{R}^{N} \times[0, T]$ to $\mathbb{R}$ i.e $u=0$ on the sides, $u>0$ inside the rectangle and $u<0$ outside (see figure 1 ). We suppose that this front evolve with a normal simplified velocity given by

$$
v\left(x_{1}, x_{2}, t\right)=\int_{K=\left\{s \in \mathbb{R}: u\left(s, x_{2}, t\right) \geq 0\right\}} d s .
$$

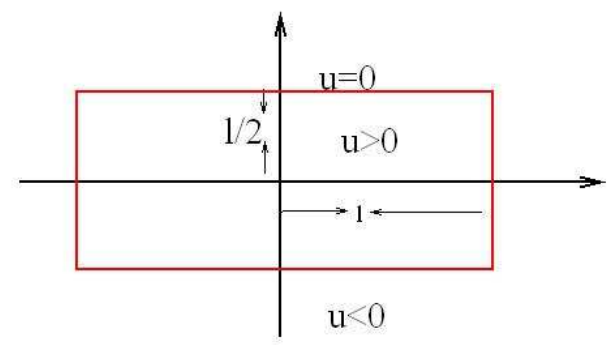

FIGURE 1. rectangular initial front

We consider now the points $\left(x, \frac{l}{2}, t\right)$ where $l$ is the width of the rectangle and $2 l$ his longer. It is easy to prove that the velocity on these points is $2 l$, but if we move vertically to $\left(x, \frac{l}{2}+\epsilon\right)$, the velocity becomes $v\left(x, \frac{l}{2}+\epsilon, t\right)=0$. 
Then, if we move vertically, a jump appears in the velocity. In the present work, instead of evolving in the normal direction, we move in the horizontal direction. Thus with the horizontally velocity, the horizontals sides of the rectangle remains fixed, whereas the vertically sides move in the horizontal direction but in two different sens. After this modification, the equation (5) becomes

$$
\left\{\begin{array}{l}
\frac{\partial u}{\partial t}=C(x, t)\left(\int_{\mathbb{R}} \mathbb{1}_{\left\{u\left(., x_{2}, t\right) \geq 0\right\}}(z) g(z) d z\right)\left|\frac{\partial u}{\partial x_{1}}\right|+\text { Trace }\left[\left(I-\frac{D u \otimes D u}{|D u|^{2}}\right) D^{2} u\right] \\
u(x, 0)=u_{0}(x) \quad \text { in } \mathbb{R}^{N} \times\{0\} .
\end{array}\right.
$$

As it was remarked by Slepčev [14, in the level-set approach, all level-sets of the solution $u$ should have the same type of normal velocity. A nonlocal term using $[u]_{x, t}^{+}$instead of $\{u(., t) \geq 0\}$ is more appropriate. It is why we consider

$\frac{\partial u}{\partial t}=C(x, t)\left(\int_{\mathbb{R}} \mathbb{1}_{\left\{u\left(., x_{2}, t\right) \geq u\left(x_{1}, x_{2}, t\right)\right\}} g(z) d z\right)\left|\frac{\partial u}{\partial x_{1}}\right|+$ Trace $\left[\left(I-\frac{D u \otimes D u}{|D u|^{2}}\right) D^{2} u\right]$.

With this modification, we will prove in Section 3 uniqueness and existence results for (7).

We now list the basic requirements on $F$. We point out that the main assumption introduced because of the presence of the nonlocal term is the monotonicity with respect to set inclusion and the continuity of $F$ with respect to the topology on $\mathscr{B}_{N-k}$ which is defined in the introduction.

(H1) $F$ is continuous from $\mathbb{R}^{N} \times[0, T] \times \mathbb{R}^{N} \backslash\{0\} \times \mathscr{S}_{N} \times \mathscr{B}_{N-k}$ into $\mathbb{R}$.

(H2) For all $\left(x, t, K^{k}, L^{k}\right) \in \mathbb{R}^{N} \times[0, T] \times \mathscr{B}_{N-k} \times \mathscr{B}_{N-k}$, we have

$$
-\infty<F_{\star}\left(x, t, 0, O, K^{k}\right)=F^{\star}\left(x, t, 0, O, L^{k}\right)<+\infty .
$$

Here $F^{\star}$ denotes the upper semicontinuous envelope of $F$, while $F_{\star}$ is the lower semicontinuous envelope of $F$. These functions are defined by

$$
\begin{aligned}
F^{\star}\left(x, t, p, X, K^{k}\right) & =\limsup _{n} F\left(x_{n}, t_{n}, p_{n}, X_{n}, K_{n}^{k}\right), \\
F_{\star}\left(x, t, p, X, K^{k}\right) & =\liminf _{n} F\left(x_{n}, t_{n}, p_{n}, X_{n}, K_{n}^{k}\right),
\end{aligned}
$$

where $\left(x_{n}, t_{n}, p_{n}, X_{n}\right)$ converges to $(x, t, p, X)$ in $\mathbb{R}^{N} \times[0, T] \times \mathbb{R}^{N} \backslash\{0\} \times \mathscr{S}_{N}$ and $K_{n}^{k}$ convergs to $K^{k}$ with respect the topology in $\mathscr{B}_{N-k}$.

(H3) $F$ is nondecreasing in its set argument i.e, for any $K^{k}, L^{k}$ in $\mathscr{B}_{N-k}$ such that $K^{k} \subset L^{k}$, we have

$$
F\left(x, t, p, X, K^{k}\right) \leq F\left(x, t, p, X, L^{k}\right)
$$

for any $X$ in $\mathscr{S}_{N}$, and for any $(x, t, p)$ in $\mathbb{R}^{N} \times[0, T] \times \mathbb{R}^{N} \backslash\{0\}$.

(H4) $F$ is geometric: for any $\lambda>0, \mu \in \mathbb{R}$, we have

$$
F\left(x, t, \lambda p, \lambda X+\mu p \otimes p, K^{k}\right)=\lambda F\left(x, t, p, X, K^{k}\right)
$$


for all $\left(x, t, p, X, K^{k}\right) \in \mathbb{R}^{N} \times[0, T] \times \mathbb{R}^{N} \backslash\{0\} \times \mathscr{S}_{N} \times \mathscr{B}_{N-k}$.

Definition 1.1. (Slepčev [14])

An upper-semicontinuous function $u: \mathbb{R}^{N} \times[0, T] \longrightarrow \mathbb{R}$, is a viscosity subsolution of (11) if, for any $\phi \in C^{2}\left(\mathbb{R}^{N} \times[0, T]\right)$, for any maximum point $(\bar{x}, \bar{t})$ of $u-\phi$, if $\bar{t}>0$ then

$$
\frac{\partial \phi}{\partial t}(\bar{x}, \bar{t}) \leq F_{\star}\left(\bar{x}, \bar{t}, D \phi(\bar{x}, \bar{t}), D^{2} \phi(\bar{x}, \bar{t}), K_{\bar{x}, \bar{t}, u}^{k,+}\right)
$$

and $u(\bar{x}, 0) \leq u_{0}(\bar{x})$ if $\bar{t}=0$.

A lower-semicontinuous function $u: \mathbb{R}^{N} \times[0, T] \longrightarrow \mathbb{R}$ is a viscosity supersolution of (11) if, for any $\phi \in C^{2}\left(\mathbb{R}^{N} \times[0, T]\right)$, for any minimum point $(\bar{x}, \bar{t})$ of $u-\phi$, if $\bar{t}>0$ then

$$
\frac{\partial \phi}{\partial t}(\bar{x}, \bar{t}) \geq F^{\star}\left(\bar{x}, \bar{t}, D \phi(\bar{x}, \bar{t}), D^{2} \phi(\bar{x}, \bar{t}), K_{\bar{x}, \bar{t}, u}^{k,-}\right)
$$

and $u(\bar{x}, 0) \geq u_{0}(\bar{x})$ if $\bar{t}=0$.

A function is a viscosity solution of (11), if it is both a subsolution and supersolution of (11).

Remark 1.1. In the definition of a subsolution and a supersolution, "test sets" were chosen differently. This is a major point when viscosity solutions are to be extended to non local, geometric parabolic equations. If " $\geq$ " were used instead of ">" in the definition of supersolutions, existence results, among other things, would hold no more. For more information, we refer the reader to the arguments given by Slepčev in [14, Definition 2.1]. For convenience, we proved another proof for these arguments in the Appendix.

In the initial compact front we will seek the solutions of our equation (the solution of which represents the front via its zero level-set) by functions in the class $\mathcal{C}$ given by the following definition.

Definition 1.2. A function $u: \mathbb{R}^{N} \times[0, T] \rightarrow \mathbb{R}$ is in the class $\mathcal{C}$, if $-1<u(x, t) \leq 1$ and $\inf _{x \in \mathbb{R}^{N}} u(x, t)=\lim _{|x| \rightarrow+\infty} u(x, t)=-1$ for all $x$ in $\mathbb{R}^{N}$ and uniformly with respect to $t \in[0, T]$.

Remark 1.2. All continuous functions in $\mathcal{C}$ are uniformly continuous bounded function and any compact front can be represented as a level-set zero of a function in $\mathcal{C}$. Indeed, let $E \subset \mathbb{R}^{N}$ be a bounded open set and $\Gamma_{0}=\partial E$ be the initial compact front. This front is represented by the signed function defined by

$$
d_{\partial E}^{s}=d\left(x, \mathbb{R}^{N} \backslash E\right)-d(x, E),
$$

where $d(x, E)$ denote the distance function to $E$. It is easy to check that $d_{\partial E}^{s}>0$ if $x \in E, d_{\partial E}^{s}<0$ if $x \in \mathbb{R}^{N} \backslash \bar{E}$ and $d_{\partial E}^{s}=0$ if and only if $x \in \Gamma_{0}$. Moreover $d_{\partial E}^{s}$ is Lipschitz continuous with constant 1 . Now, we consider the function $f(x)=2 \arctan \left(d_{\partial E}^{s}(x)\right) / \pi$ and we use the properties of the arctan function to prove that $f(x)>-1$ for all $x \in \mathbb{R}^{N}$ and $f(x)$ tends to -1 as $x$ tends to $\infty$. Then, $f \in \mathcal{C}$ and the initial compact front $\Gamma_{0}$ 
is represented by $f$. A typical example of function which belongs to this class is $g(x, t)=\frac{e^{-A t}}{1+|x|^{2}}-1$ where $(x, t) \in \mathbb{R}^{N} \times[0, T]$ and $A$ is a positive constant.

Now, another question comes out: for which reason, we use the class $\mathcal{C}$ for sub and supersolutions in the compact front case? This class $\mathcal{C}$ allows to treat the sets $K_{x, t, u}^{k, \pm}$ of infinite volume. To explain that, we start with an initial front which propagates with the following velocity

$$
V\left(x, t,[u]_{x, t}^{+}\right)=\left(\int_{[u]_{x, t}^{+}} d z\right)^{\frac{1}{N}}=(\operatorname{vol}\{u(., t) \geq u(x, t)\})^{\frac{1}{N}} .
$$

When we describe this evolution by the level-set method we obtain the nonlocal level-set equation

$$
\left\{\begin{array}{l}
\frac{\partial u}{\partial t}(x, t)=(\operatorname{vol}\{u(., t) \geq u(x, t)\})^{\frac{1}{N}}|D u| \quad \text { in } \mathbb{R}^{N} \times[0, T], \\
u(x, 0)=u_{0}(x) \quad \text { in } \mathbb{R}^{N} \times\{0\} .
\end{array}\right.
$$

First, the power $\frac{1}{N}$ ensures the existence of a viscosity solution of (8) and ensures that the front does not explode on short time $t$ (see [6, Remark 4.1]). Moreover, if the solution $u$ belongs to the class $\mathcal{C}$, for any $(x, t) \in \mathbb{R}^{N} \times[0, T]$ there exists $M_{x, t}$ such that, the Lebesgue measure $\mathcal{L}^{N}\left([u]_{x, t}\right) \leq M_{x, t}<\infty$. Whence, the previous definition of viscosity solution remains valid for the equation (8).

\section{UNIQUENESS RESULT FOR COMPACT FRONTS}

In the case of compact fronts, we start with a compact initial front and we prove that the front remains compact i.e. there exists a solution $u$ of (11) which lies in $\mathcal{C}$. We use the following assumptions:

(H0) Initial compact front: $u_{0} \in \mathrm{C}\left(\mathbb{R}^{N}\right)$ and $u_{0} \in \mathcal{C}$. Now, for $\theta \in\{0,1\}$, we have the following assumptions.

(H5- $\theta$ ) There exist positive constants $L_{1}$ and $L_{2}$ such that for $\left(x, t, p, X, K^{k}\right)$ in $\mathbb{R}^{N} \backslash\{0\} \times[0, T] \times \mathbb{R}^{N} \times \mathscr{S}_{N} \times \mathscr{B}_{N-k}$, we have

$\left|F\left(x, t, p, X, K^{k}\right)\right| \leq L_{1}\left((1+|x|)|p|+\theta \mathcal{L}^{N-k}\left(K^{k}\right)^{\frac{1}{N-k}}\left|\tilde{\pi}_{k}(p)\right|\right)+L_{2}\left(1+|x|^{2}\right)|X|_{\infty}$

where $|A|_{\infty}=\max _{1 \leq i \leq N} \sum_{j=1}^{N}\left|a_{i, j}\right|$, for any matrix $A=\left(a_{i, j}\right)_{1 \leq i, j \leq N}$ in $\mathscr{S}_{\mathrm{N}}$ and $\tilde{\pi}_{k}$ denote the projection from $\mathbb{R}^{N}$ into $\mathbb{R}^{N-k}$ defined by $\tilde{\pi}_{k}(x)=$ $\left(x_{k+1}, \ldots, x_{N}\right)=x-\pi_{k}(x)$, for all $x \in \mathbb{R}^{N}$. 
(H6- $\theta$ ) There exists a nondecreasing modulus of continuity $w:[0, \infty] \rightarrow$ $[0, \infty]$ which satisfies $w(0+)=0$ and

$F\left(x, t, p, X, K^{k}\right)-F\left(y, t, p, Y, K^{k}\right) \leq w\left(|x-y|\left(1+|p|+\theta \mathcal{L}\left(K^{k}\right)^{\frac{1}{N-K}}\left|\tilde{\pi}_{k}(p)\right|\right)+\sigma|x-y|^{2}\right)$

whenever $\left(x, y, t, p, K^{k}\right) \in \mathbb{R}^{N} \times \mathbb{R}^{N} \times[0, T] \times \mathbb{R}^{N} \backslash\{0\} \times \mathscr{B}_{N-k}, \sigma$ is a positive constant and $X, Y$ satisfying the inequality $\left(\begin{array}{cc}X & 0 \\ 0 & -Y\end{array}\right) \leq\left(\begin{array}{cc}Z & -Z \\ -Z & Z\end{array}\right)$, where $Z \in \mathscr{S}_{N}$ and $\leq$ stands for the partial ordering in $\mathscr{S}_{N}$.

(H7) For $1<k \leq N$, for all $p$ in $\mathbb{R}^{N}$, there exists a continuous function $G$ from $\mathbb{R}^{\mathbb{N}} \times[0, T] \times \mathbb{R}^{k} \backslash\{0\} \times \mathscr{S}_{N}$ to $\mathbb{R}^{N-k}$ such that: if $\tilde{\pi}_{k}(p)=0$ then

$$
F\left(x, t, p, X, K^{k}\right)=G\left(x, t, \pi_{k}(p), X\right)
$$

for all $(x, t, p, X) \in \mathbb{R}^{N} \times[0, T] \times \mathbb{R}^{N} \backslash\{0\} \times \mathscr{S}_{N}$. Moreover, we suppose that, $G$ satisfies the assumption (H6-0).

Theorem 2.1. Assume (H0)-(H1)-(H2)-(H3)-(H4)-(H5)-(H6-1) and (H7). Let $u \in \mathcal{C}$ (resp. $v \in \mathcal{C}$ ) be a bounded upper-semicontinous subsolution of (1) (resp. bounded lower-semicontinous supersolution of (1)), then $u \leq v$ in $\mathbb{R}^{N} \times[0, T]$.

Corollary 2.1. Under the assumptions of Theorem 2.1, there exists a unique viscosity solution $u \in \mathcal{C}$ of (11).

The proof of this Corollary is postponed to (Section 5).

\section{Proof of Theorem 2.1.}

1. The test-function. We argue by contradiction assuming there exists $(\hat{x}, \hat{t}) \in \mathbb{R}^{N} \times[0, T]$ such that

$$
u(\hat{x}, \hat{t})-v(\hat{x}, \hat{t})=M>0 .
$$

Since $u$ and $v$ are bounded, the following supremum

$$
M_{\epsilon, \eta}=\sup _{(x, y, t) \in\left(\mathbb{R}^{N}\right)^{2} \times[0, T]}\left\{u(x, t)-v(y, t)-\sum_{i=0}^{N} \frac{\left|x_{i}-y_{i}\right|^{4}}{4 \epsilon^{2}}-\eta t\right\}
$$

is finite for any $\epsilon, \eta>0$. We choose $\eta$ is small enough so that

$$
M_{\epsilon, \eta} \geq \frac{M}{2}>0 \text {. }
$$

Since $u, v \in \mathcal{C}$, we have

$$
\lim _{|x| \rightarrow+\infty}[u(x, t)-v(x, t)]=0
$$

uniformly with respect to $t \in[0, T]$. Therefore from (10), the supremum in (9) is achieved at a point $(\bar{x}, \bar{y}, \bar{t})$,

$$
M_{\epsilon, \eta}=u(\bar{x}, \bar{t})-v(\bar{y}, \bar{t})-\sum_{i=1}^{N} \frac{\left|\bar{x}_{i}-\bar{y}_{i}\right|^{4}}{4 \epsilon^{4}}-\eta \bar{t} .
$$


Actually $\bar{x}, \bar{y}$ and $\bar{t}$ depend on $\epsilon, \eta$, but we omit this dependence in the notation for simplicity.

2. Viscosity inequalities when $\bar{t}>0$. From the fundamental result of the User's guide to viscosity solutions [9, Theorem 8.3], for every $\rho>0$, we get $a_{1}, a_{2} \in \mathbb{R}$ and $\bar{X}, \bar{Y} \in \mathscr{S}_{N}$ such that

$$
\left(a_{1}, \bar{p}, \bar{X}\right) \in \overline{\mathcal{P}}^{2,+} u(\bar{x}, \bar{t}), \quad\left(a_{2}, \bar{p}, \bar{Y}\right) \in \overline{\mathcal{P}}^{2,-} v(\bar{y}, \bar{t})
$$

and

$$
\begin{gathered}
-\frac{1}{\rho}\left(\begin{array}{cc}
I & 0 \\
0 & I
\end{array}\right) \leq\left(\begin{array}{cc}
\bar{X} & 0 \\
0 & -\bar{Y}
\end{array}\right) \leq\left(\begin{array}{cc}
Z+\rho Z^{2} & -\left(Z+\rho Z^{2}\right) \\
-\left(Z+\rho Z^{2}\right) & Z+\rho Z^{2}
\end{array}\right) \\
a_{1}-a_{2}=\eta
\end{gathered}
$$

for $Z=D^{2} \varphi(\bar{x}-\bar{y})$, where $\varphi(x-y)=\sum_{i=0}^{N} \frac{\left|x_{i}-y_{i}\right|^{4}}{4 \epsilon^{2}}, I$ is the identity matrix and

$$
\bar{p}=\left(\frac{\left|\bar{x}_{1}-\bar{y}_{1}\right|^{2}\left(\bar{x}_{1}-\bar{y}_{1}\right)}{\epsilon^{4}}, \cdots, \frac{\left|\bar{x}_{N}-\bar{y}_{N}\right|^{2}\left(\bar{x}_{N}-\bar{y}_{N}\right)}{\epsilon^{4}}\right) .
$$

Writing that $u$ is a subsolution and $v$ a supersolution of (11), we have

$$
\eta \leq F_{\star}\left(\bar{x}, \bar{t}, \bar{p}, \bar{X}, K_{\bar{x}, \bar{t}, u}^{k,+}\right)-F^{\star}\left(\bar{y}, \bar{t}, \bar{p}, \bar{Y}, K_{\bar{y}, \bar{t}, v}^{k,-}\right) .
$$

Remark 2.1. The reader should note a difficulty in applying [9, Theorem 8.3] here. Indeed, one should double the time variable to prove [9, Theorem 8.3]. It is not straightforward here because of the presence of the nonlocal term. This problem is solved by the stability result provided in [14]. However, we give another proof of the stability in the appendix .

3. Comparison of the nonlocal terms . From the definition of $M_{\epsilon, \eta}$, for all $(x, y) \in \mathbb{R}^{N} \times \mathbb{R}^{N}$, we have

$u(x, \bar{t})-v(y, \bar{t})-\sum_{i=1}^{N} \frac{\left|x_{i}-y_{i}\right|^{4}}{4 \epsilon^{4}} \leq u(\bar{x}, \bar{t})-v(\bar{y}, \bar{t})-\sum_{i=1}^{N} \frac{\left|\bar{x}_{i}-\bar{y}_{i}\right|^{4}}{4 \epsilon^{4}}$.

Taking $x=\left(\pi_{k}(\bar{x}), z\right)$ and $y=\left(\pi_{k}(\bar{y}), z\right)$, for any $z \in \mathbb{R}^{N-k}$, we have

$u\left(\pi_{k}(\bar{x}), z, \bar{t}\right)-v\left(\pi_{k}(\bar{y}), z, \bar{t}\right)-\sum_{i=1}^{k} \frac{\left|\bar{x}_{i}-\bar{y}_{i}\right|^{4}}{4 \epsilon^{4}} \leq u(\bar{x}, \bar{t})-v(\bar{y}, \bar{t})-\sum_{i=1}^{N} \frac{\left|\bar{x}_{i}-\bar{y}_{i}\right|^{4}}{4 \epsilon^{4}}$,

thus we obtain

$$
u\left(\pi_{k}(\bar{x}), z, \bar{t}\right)-u(\bar{x}, \bar{t}) \leq v\left(\pi_{k}(\bar{y}), z, \bar{t}\right)-v(\bar{y}, \bar{t})-\sum_{i=k+1}^{N} \frac{\left|\bar{x}_{i}-\bar{y}_{i}\right|^{4}}{4 \epsilon^{4}} .
$$

4. Upper-bound for the volume $K_{\bar{x}, \bar{t}, u}^{k,+}$ and conclusion.

Since $u, v \in \mathcal{C}$ and by (10) and (11), $\bar{x}$ and $\bar{y}$ remain bounded independently of $\epsilon$ and $\eta$. Using $v \in \mathcal{C}$, we write

$$
\lim _{\epsilon, \eta \rightarrow 0} v(\bar{y}, \bar{t})>-1
$$


then, there exist a positive constant $\mu$ independent of $\epsilon$ and $\eta$, such that $v(\bar{y}, \bar{t}) \geq-1+\mu$. Then

$$
K_{\bar{y}, \bar{t}, v}^{k,+} \subset\left\{z \in \mathbb{R}^{N-k}: v\left(\pi_{k}(\bar{y}), z, \bar{t}\right) \geq-1+\mu\right\} \subset \overline{B(0, R)}
$$

where $R$ is a positive constant independent of $\epsilon$ and $\eta$. By the inequality (13), if $u\left(\pi_{k}(\bar{x}), z, \bar{t}\right)-u(\bar{x}, \bar{t}) \geq 0$, we have $v\left(\pi_{k}(\bar{y}), z, \bar{t}\right)-v(\bar{y}, \bar{t}) \geq 0$, then

$$
K_{\bar{x}, \bar{t}, u}^{k,+} \subset K_{\bar{y}, \bar{t}, v}^{k,+} \subset \overline{B(0, R)} .
$$

We distinguish two cases:

First case: $\bar{x}_{i}=\bar{y}_{i}$ for all $k<i \leq N$. In this case we have

$$
\bar{p}=\left(\frac{\left|\bar{x}_{1}-\bar{y}_{1}\right|^{2}\left(\bar{x}_{1}-\bar{y}_{1}\right)}{\epsilon^{4}}, \cdots, \frac{\left|\bar{x}_{k}-\bar{y}_{k}\right|^{2}\left(\bar{x}_{k}-\bar{y}_{k}\right)}{\epsilon^{4}}, 0, \cdots, 0\right) .
$$

In other words, $\tilde{\pi}_{k}(\bar{p})=0$. First, if $\bar{x}_{i}=\bar{y}_{i}$ for any $1 \leq i \leq k$ then $\bar{p}=0$. But, by [9, Theorem 3.2], and [5, Lemma 2.4.3], there exist $\bar{X}^{\prime}, \bar{Y}^{\prime} \in \mathscr{S}^{N}$ such that

$$
\bar{X} \leq \bar{X}^{\prime} \leq \bar{Y}^{\prime} \leq \bar{Y},
$$

with $\bar{X}^{\prime}=\bar{Y}^{\prime}=0$ when $\bar{p}=0$. Taking advantage of the ellipticity of $F^{\star}$ and $F_{\star}$, we get from (12)

$$
\eta \leq F_{\star}\left(\bar{x}, \bar{t}, 0, O, K_{\bar{x}, \bar{t}, u}^{k,+}\right)-F^{\star}\left(\bar{x}, \bar{t}, 0, O, K_{\bar{y}, \bar{t}, v}^{k,-}\right) .
$$

Assumption (H2) implies that

$$
\eta \leq 0
$$

which is a contradiction since $\eta>0$.

Therefore, there exists $1 \leq i_{0} \leq k$ such that $\bar{x}_{i_{0}} \neq \bar{y}_{i_{0}}$ and $\bar{p} \neq 0$. Using (H7) and the viscosity inequality in (12), we get

$$
\eta \leq G\left(\bar{x}, \bar{t}, \pi_{k}(\bar{p}), \bar{X}\right)-G\left(\bar{y}, \bar{t}, \pi_{k}(\bar{p}), \bar{Y}\right) .
$$

By (H7), $G$ satisfies the assumptions (H6-0) then

$$
\begin{aligned}
\eta & \leq w\left(|\bar{x}-\bar{y}|\left(1+\left|\pi_{k}(\bar{p})\right|\right)+\sigma|\bar{x}-\bar{y}|^{2}\right) \\
& \leq w\left(|\bar{x}-\bar{y}|(1+|\bar{p}|)+\sigma|\bar{x}-\bar{y}|^{2}\right) .
\end{aligned}
$$

Since $|\bar{p}|<\frac{|\bar{x}-\bar{y}|^{3}}{4 \epsilon^{4}}$, then $|\bar{x}-\bar{y}||\bar{p}| \leq \frac{|\bar{x}-\bar{y}|^{4}}{4 \epsilon^{4}}$. From this estimate, the inequality in (15) becomes

$$
\eta \leq w\left(|\bar{x}-\bar{y}|+\frac{|\bar{x}-\bar{y}|^{4}}{4 \epsilon^{2}}+\sigma|\bar{x}-\bar{y}|^{2}\right) .
$$

Classical arguments [9, Remark 3.8] show that $|\bar{x}-\bar{y}|$ and the penalisation term $\sum_{i=1}^{N} \frac{\left|\bar{x}_{i}-\bar{y}_{i}\right|^{4}}{4 \epsilon^{2}}$ tends to 0 if $\epsilon$ tends to 0 , then $\frac{|\bar{x}-\bar{y}|^{4}}{4 \epsilon^{2}}$ tends to 0 if $\epsilon$ tends to zero. Finally we let $\epsilon$ tend to 0 in (15), to obtain $\eta \leq 0$ which is a absurd. 
Second case: There exists $i_{0}$ such that $k<i_{0} \leq N$ and $\bar{x}_{i_{0}} \neq \bar{y}_{i_{0}}$. In this case, for all $z \in \mathbb{R}^{N-k}$, we have from (13)

$$
\begin{aligned}
u\left(\pi_{k}(\bar{x}), z, \bar{t}\right)-u(\bar{x}, \bar{t}) & \leq v\left(\pi_{k}(\bar{y}), z, \bar{t}\right)-v(\bar{y}, \bar{t})-\frac{\left|\bar{x}_{i_{0}}-\bar{y}_{i_{0}}\right|^{4}}{4 \epsilon^{2}} \\
& <v\left(\pi_{k}(\bar{y}), z, \bar{t}\right)-v(\bar{y}, \bar{t}) .
\end{aligned}
$$

and therefore

$$
K_{\bar{x}, \bar{t}, u}^{k,+} \subset K_{\bar{y}, \bar{t}, v}^{k,-}
$$

Since $\bar{p} \neq 0$ in this case, the inequality viscosity in (12) becomes

$$
\eta \leq F\left(\bar{x}, \bar{t}, \bar{p}, \bar{X}, K_{\bar{x}, \bar{t}, u}^{k,+}\right)-F\left(\bar{y}, \bar{t}, \bar{p}, \bar{Y}, K_{\bar{x}, \bar{t}, v}^{k,-}\right) .
$$

From (H3) and (17), we get

$$
\eta \leq F\left(\bar{x}, \bar{t}, \bar{p}, \bar{X}, K_{\bar{x}, \bar{t}, u}^{k,+}\right)-F\left(\bar{y}, \bar{t}, \bar{p}, \bar{Y}, K_{\bar{x}, \bar{t}, u}^{k,+}\right) .
$$

We use the assumption (H6-1) to write

$$
\left.\eta \leq w\left(|\bar{x}-\bar{y}|\left(1+|\bar{p}|+\mathcal{L}^{N-k}\left(K_{\bar{x}, \bar{t}, u}^{k,+}\right)^{\frac{1}{N-k}}\left|\tilde{\pi}_{k}(\bar{p})\right|+\sigma|\bar{x}-\bar{y}|^{2}\right) \mid\right)\right) .
$$

Now, using the estimate in (14), we have

$$
\mathcal{L}^{N-k}\left(K_{\bar{x}, \bar{t}, u}^{k,+}\right)^{\frac{1}{N-k}} \leq \mathcal{L}^{N-k}(\overline{B(0, R)})^{\frac{1}{N-k}} \leq C_{N-k} R
$$

where $C_{N-k}=\mathcal{L}^{N-k}\left(B_{N-k}(0,1)\right)^{\frac{1}{N-k}}$, and $R$ is independent of $\epsilon$. Then

$$
\begin{aligned}
\eta & \leq w\left(|\bar{x}-\bar{y}|\left(1+|\bar{p}|+C_{N-k} R\left|\tilde{\pi}_{k}(\bar{p})\right|\right)+\sigma|\bar{x}-\bar{y}|^{2}\right) \\
& \leq w\left(|\bar{x}-\bar{y}|+\frac{|\bar{x}-\bar{y}|^{4}}{4 \epsilon^{2}}+C_{N-k} R \frac{|\bar{x}-\bar{y}|^{4}}{4 \epsilon^{2}}+\sigma|\bar{x}-\bar{y}|^{2}\right) .
\end{aligned}
$$

Since we know that $\frac{|\bar{x}-\bar{y}|^{4}}{4 \epsilon^{2}}$ and therefore $|\bar{x}-\bar{y}|$ converges to 0 as $\epsilon$ goes to 0 , the above inequality (19) implies that $\eta \leq 0$ which is a contradiction since $\eta>0$.

6. The case $\bar{t}=0$. From the above step we obtain that the maximum $M_{\epsilon, \eta}$ is achieved for $\bar{t}=0$. From (10) and using the uniform continuity of $u_{0}\left(u_{0} \in \mathcal{C} \cap C\left(\mathbb{R}^{N}\right)\right)$, for any $\rho \geq 0$, there exists $L_{\rho}>0$ such that

$$
\frac{M}{2} \leq M_{\epsilon, \eta}=u_{0}(\bar{x})-u_{0}(\bar{y})-\sum_{i=1}^{N} \frac{\left|\bar{x}_{i}-\bar{y}_{i}\right|^{4}}{4 \epsilon^{2}} \leq \rho+L_{\rho}|\bar{x}-\bar{y}| \leq \rho+\frac{L_{\rho}^{2} \epsilon^{2}}{4}
$$

which leads to a contradiction taking $\rho<\frac{M}{2}$ and sending $\epsilon$ to 0 . 


\section{UNIQUENESS RESULT FOR NON COMPACT FRONTS}

In the previous section we considered the assumption (H0), this assumption forced us to deal with a compact initial front $\Gamma_{0}=\left\{u_{0}=0\right\}$. In this section we deal with non compact fronts. Instead of (H0), we consider the assumptions:

(H0') Non compact initial front : $u_{0} \in B U C\left(\mathbb{R}^{N}\right)$.

(H1') For all $(p, X) \in \mathbb{R}^{N} \times \mathscr{S}_{N}$, we have

$$
\lim _{|p|,|X| \rightarrow 0} F^{\star}\left(x, t, p, X, K^{k}\right)=\lim _{|p|,|X| \rightarrow 0} F_{\star}\left(x, t, p, X, K^{k}\right)=0,
$$

uniformly with respect to $\left(x, t, K^{k}\right) \in \mathbb{R}^{N} \times[0, T] \times \mathscr{B}_{N-k}$.

(H2') For all $\left(x, t, K^{k}\right) \in \mathbb{R}^{N} \times[0, T] \times \mathscr{B}_{N-k}$ and for all $(p, X, Y)$ in $\mathbb{R}^{N} \backslash\{0\} \times \mathscr{S}_{N} \times \mathscr{S}_{N}$ such that $(|p|+|X|),(|p|+|Y|) \leq R$, where $R$ is a positive constant, There exists a nondecreasing modulus of continuity $w_{R}: \mathbb{R}_{+} \longrightarrow \mathbb{R}_{+}$and $w_{R}\left(0^{+}\right)=0$ such that

$$
F\left(x, t, p, X, K^{k}\right)-F\left(x, t, q, Y, K^{k}\right) \leq w_{R}\left(\left(|X-Y|_{\infty}+|p-q|\right)(1+|x|)\right) .
$$

(H3') For any $K^{k}, L^{k} \in \mathscr{B}_{N-k}$ such that $K^{k} \backslash L^{k} \subset \mathbb{R}^{N-k} \backslash B(0, r)$, where $r>0$, and for bounded $(p, X) \in \mathbb{R}^{N} \times \mathscr{S}_{N}$ we have

$$
F\left(x, t, p, X, K^{k}\right)-F\left(x, t, p, X, L^{k}\right) \rightarrow 0
$$

if $r$ tends to $+\infty$ uniformly with respect to $(x, t) \in \mathbb{R}^{N} \times[0, T]$.

(H4') For any $K^{k}, L^{k} \in \mathscr{B}_{N-k}$, for any bounded $(p, X) \in \mathbb{R}^{N} \backslash\{0\} \times \mathscr{S}_{N}$ such that $\left|\tilde{\pi}_{k}(p)\right| \leq \lambda$, we have

$$
F\left(x, t, p, X, K^{k}\right)-F\left(x, t, p, X, L^{k}\right) \rightarrow 0
$$

if $\lambda$ tends to 0 uniformly with respect to $(x, t) \in \mathbb{R}^{N} \times[0, T]$.

Remark 3.1. The assumption (H3') implies that, if the Lebesgue measure of $K^{k} \backslash L^{k}$ is negligible, then the difference between $F\left(x, t, p, X, K^{k}\right)$ and $F\left(x, t, q, X, L^{k}\right)$ is small for any $x \in \mathbb{R}^{N}$ and $X$ bounded and uniformly with respect to $t \in[0, T]$. In addition, since we deal with the non compact front (H0'), we cannot control the nonlocal terms and instead of the assumption (H5-1), we consider the assumption (H5-0).

Theorem 3.1. Assume (H0')- (H1')-(H2')-(H3')-(H4')-(H4)-(H5-0)(H6-0). Let u (resp. $v$ ) be a bounded upper-semicontinuous subsolution of (11) (resp. bounded lower semicontinuous supersolution of (11)), then $u \leq v$ in $\mathbb{R}^{N} \times[0, T]$.

Corollary 3.1. Under the assumptions of Theorem 3.1, there exists a unique viscosity solution $u$ of (1). 
The demonstration of this Corollary is postponed to (Section 5).

\section{Proof of the Theorem 3.1}

1. The test-function. We argue by contradiction. We suppose that there exists $(\tilde{x}, \tilde{t})$ such that

$$
0<M=u(\tilde{x}, \tilde{t})-v(\tilde{x}, \tilde{t}) .
$$

In this case, we have to add some terms in the test-function in order to deal with non compact front. We consider

$$
M_{\epsilon, \eta, \alpha}=\sup _{\mathbb{R}^{N} \times \mathbb{R}^{N} \times[0, T]}\left\{u(x, t)-v(y, t)-\left(\sum_{i=1}^{N} \frac{\left|x_{i}-y_{i}\right|^{4}}{4 \epsilon^{2}}+\alpha|x|^{2}+\alpha|y|^{2}\right)-\eta t\right\} .
$$

Since $u, v$ are bounded, for $\epsilon, \alpha, \eta>0$, the supremum is achieved at a point $(\bar{x}, \bar{y}, \bar{t})$ and for $\alpha, \eta$ are small enough, we have

$$
M_{\epsilon, \eta, \alpha} \geq \frac{M}{2}>0 \text {. }
$$

2. Viscosity inequalities when $\bar{t}>0$. From the fundamental result of the User's guide to viscosity solutions [9, Theorem 8.3], for every $\rho>0$, we get $a_{1}, a_{2} \in \mathbb{R}$ and $\bar{X}, \bar{Y} \in \mathscr{S}^{N}$ such that

$\left(a_{1}, \bar{p}+2 \alpha \bar{x}, \bar{X}+2 \alpha I\right) \in \overline{\mathcal{P}}^{2,+} u(\bar{x}, \bar{t}), \quad\left(a_{2}, \bar{p}-2 \alpha \bar{y}, \bar{Y}-2 \alpha I\right) \in \overline{\mathcal{P}}^{2,-} v(\bar{y}, \bar{t})$,

$$
a_{1}-a_{2}=\eta
$$

and

$$
\frac{-1}{\rho}\left(\begin{array}{cc}
I & 0 \\
0 & I
\end{array}\right) \leq\left(\begin{array}{cc}
\bar{X} & 0 \\
0 & -\bar{Y}
\end{array}\right) \leq\left(\begin{array}{cc}
Z+\rho Z^{2} & -\left(Z+\rho Z^{2}\right) \\
-\left(Z+\rho Z^{2}\right) & Z+\rho Z^{2}
\end{array}\right)
$$

for $Z=D^{2} \varphi(\bar{x}-\bar{y})$, where $\varphi(x-y)=\sum_{i=0}^{N} \frac{\left|x_{i}-y_{i}\right|^{4}}{4 \epsilon^{2}}$ for any $(x, y) \in \mathbb{R}^{N} \times \mathbb{R}^{N}$ and

$$
\bar{p}=\left(\frac{\left|\bar{x}_{1}-\bar{y}_{1}\right|^{2}\left(\bar{x}_{1}-\bar{y}_{1}\right)}{\epsilon^{4}}, \cdots, \frac{\left|\bar{x}_{N}-\bar{y}_{N}\right|^{2}\left(\bar{x}_{N}-\bar{y}_{N}\right)}{\epsilon^{4}}\right) .
$$

Moreover, by [9, Remark 3.8] and [11, Proposition 2.5], $|\bar{X}|_{\infty},|\bar{Y}|_{\infty}$ and $|\bar{x}-\bar{y}|$ are bounded (independently of $\alpha$ ) and we have

$$
\begin{gathered}
\lim _{\epsilon \rightarrow 0} \frac{|\bar{x}-\bar{y}|^{4}}{4 \epsilon^{2}}=0, \lim _{\epsilon, \alpha \rightarrow 0} \alpha\left(|\bar{x}|^{2}+|\bar{y}|^{2}\right)=0, \\
\lim _{\alpha \rightarrow 0} \alpha|\bar{y}|=\lim _{\alpha \rightarrow 0} \alpha|\bar{x}|=0 .
\end{gathered}
$$

Writing that $u$ is a subsolution and $v$ a supersolution of (11), we have

$$
\eta \leq F_{\star}\left(\bar{x}, \bar{t}, \bar{p}+2 \alpha \bar{x}, \bar{X}+2 \alpha I, K_{\bar{x}, \bar{t}, u}^{k,+}\right)-F^{\star}\left(\bar{y}, \bar{t}, \bar{p}-\alpha \bar{y}, \bar{Y}-2 \alpha I, K_{\bar{y}, \bar{t}, v}^{k,-}\right) .
$$

3. Difference between $K_{\bar{x}, \bar{t}, u}^{k,+}$ and $K_{\bar{y}, \bar{t}, v}^{k,-}$. Because of the presence of term $\alpha|x|^{2}+\alpha|y|^{2}$ in the test function, the procedure used in the compact front 
case (Step 4) is non valid. Now, since $(\bar{x}, \bar{y}, \bar{t})$ is the maximum point of (20), we have

$$
\begin{aligned}
& u\left(\pi_{k}(\bar{x}), z, \bar{t}\right)-v\left(\pi_{k}(\bar{y}), z, \bar{t}\right) \\
& \leq u(\bar{x}, \bar{t})-v(\bar{y}, \bar{t})-\sum_{i=k+1}^{N}\left(\frac{\left|\bar{x}_{i}-\bar{y}_{i}\right|^{4}}{4 \epsilon^{4}}+\alpha\left(\bar{x}_{i}^{2}+\bar{y}_{i}^{2}\right)\right)+2 \alpha|z|^{2} .
\end{aligned}
$$

From the definition of $K_{\bar{x}, \bar{t}, u}^{k,+}$ and $K_{\bar{y}, \bar{t}, v}^{k,-}$, we have

$$
K_{\bar{x}, \bar{t}, u}^{k,+} \subset K_{\bar{y}, \bar{t}, v}^{k,-} \cup \overline{\mathcal{E}}
$$

where $\overline{\mathcal{E}}=K_{\bar{x}, \bar{t}, u}^{k,+} \cap\left\{v\left(\pi_{k}(\bar{y}), \cdot, \bar{t}\right) \leq v(\bar{x}, \bar{t})\right\}$. If $z \in \overline{\mathcal{E}}$, we have

$$
u(\bar{x}, \bar{t})-v(\bar{y}, \bar{t}) \leq u\left(\pi_{k}(\bar{x}), z, \bar{t}\right)-v\left(\pi_{k}(\bar{y}), z, \bar{t}\right) .
$$

It follows that

$$
\overline{\mathcal{E}} \subset\left\{z \in \mathbb{R}^{N-k}:|z|^{2} \geq \frac{1}{2} \sum_{i=k+1}^{N}\left(\frac{\left|\bar{x}_{i}-\bar{y}_{i}\right|^{4}}{4 \alpha \epsilon^{4}}+\bar{x}_{i}^{2}+\bar{y}_{i}^{2}\right)\right\} .
$$

4. Estimate of the term hand and side of the inequality (23). We distinguish two cases :

First case: $\lim _{\alpha \rightarrow 0} \bar{p}=0$. By [9, Theorem 3.8] we have $\bar{X} \leq D_{\bar{x} \bar{x}}^{2} \varphi(\bar{x}, \bar{y}, \bar{t})$ and $\bar{Y} \geq-D_{\bar{y} \bar{y}}^{2} \varphi(\bar{x}, \bar{y}, \bar{t})$. Taking advantage of the ellipticity of $F^{\star}$ and $F_{\star}$ in the viscosity inequality (23), we obtain

$\eta \leq F_{\star}\left(\bar{x}, \bar{t}, \bar{p}+2 \alpha \bar{x}, D_{\bar{x} \bar{x}}^{2} \varphi(\bar{x}, \bar{y}, \bar{t})+2 \alpha I, K_{\bar{x}, \bar{t}, u}^{k,+}\right)-F^{\star}\left(\bar{y}, \bar{t}, \bar{p}-\alpha \bar{y},-D_{\bar{y} \bar{y}}^{2} \varphi(\bar{x}, \bar{y}, \bar{t})-2 \alpha I, K_{\bar{y}, \bar{t}, v}^{k,-}\right)$.

But, since $\lim _{\alpha \rightarrow 0} \bar{p}=0$, then $\lim _{\alpha \rightarrow 0} D_{\bar{x}}^{2} \varphi(\bar{x}, \bar{y}, \bar{t})=0$ and $\lim _{\alpha \rightarrow 0} D_{\bar{y}}^{2} \varphi(\bar{x}, \bar{y}, \bar{t})=0$. We send $\alpha$ to zero and we use the assumption (H1') to write $\eta \leq 0$, which is a contradiction since $\eta>0$.

Second case: $\lim _{\alpha \rightarrow 0} \bar{p} \neq 0$. In which case, up to extract a subsequence there exists $\nu>0$ such that $\bar{p} \geq \nu$. Now, by (22) we can suppose that $\bar{p} \neq 0, \bar{p}+2 \alpha \bar{x} \neq 0, \bar{p}-2 \alpha \bar{y} \neq 0$ and we have the following estimate $F\left(\bar{x}, \bar{t}, \bar{p}+2 \alpha \bar{x}, \bar{X}+2 \alpha I, K_{\bar{x}, \bar{t}, u}^{k,+}\right)-F\left(\bar{y}, \bar{t}, \bar{p}-2 \alpha \bar{y}, \bar{Y}-2 \alpha I, K_{\bar{x}, \bar{t}, v}^{k,-}\right)$ $\leq\left(I_{1}\right)+\left(I_{2}\right)+\left(I_{3}\right)+\left(I_{4}\right)$, where

$$
\begin{aligned}
& \left(I_{1}\right)=F\left(\bar{x}, \bar{t}, \bar{p}+2 \alpha \bar{x}, \bar{X}+2 \alpha I, K_{\bar{x}, \bar{t}, u}^{k,+}\right)-F\left(\bar{x}, \bar{t}, \bar{p}, \bar{X}, K_{\bar{x}, \bar{t}, u}^{k,+}\right), \\
& \left(I_{2}\right)=F\left(\bar{x}, \bar{t}, \bar{p}, \bar{X}, K_{\bar{x}, \bar{t}, u}^{k,+}\right)-F\left(\bar{y}, \bar{t}, \bar{p}, \bar{Y}, K_{\bar{x}, \bar{t}, u}^{k,+}\right), \\
& \left(I_{3}\right)=F\left(\bar{y}, \bar{t}, \bar{p}, \bar{Y}, K_{\bar{x}, \bar{t}, u}^{k,+}\right)-F\left(\bar{y}, \bar{t}, \bar{p}, \bar{Y}, K_{\bar{y}, \bar{t}, v}^{k,-}\right), \\
& \left(I_{4}\right)=F\left(\bar{y}, \bar{t}, \bar{p}, \bar{Y}, K_{\bar{y}, \bar{t}, v}^{k,-}\right)-F\left(\bar{y}, \bar{t}, \bar{p}-2 \alpha \bar{y}, \bar{Y}-2 \alpha I, K_{\bar{y}, \bar{t}, v}^{k,-}\right) .
\end{aligned}
$$

By the classical argument in [9, Theorem 8.3] and [11, Proposition 2.5], $\bar{p}+2 \alpha \bar{x}, \bar{X}, \bar{Y}$ and $\bar{p}+2 \alpha \bar{x}$ are bounded independently of $\alpha$. We suppose that $R_{\epsilon}=\operatorname{Max}((|\bar{p}+2 \alpha \bar{x}|+|\bar{X}|),(|\bar{p}-2 \alpha \bar{y}|+|\bar{Y}|))$, and we use the assumption 
(H2'), to obtain

$$
\begin{aligned}
\left(I_{1}\right) & \leq w_{R_{\epsilon}}\left(\left(2 \alpha|I|_{\infty}+2 \alpha|\bar{x}|\right)(1+|\bar{x}|)\right) \\
& \leq w_{R_{\epsilon}}\left(2 \alpha+4 \alpha|\bar{x}|+2 \alpha|\bar{x}|^{2}\right)
\end{aligned}
$$

since $|I|_{\infty}=1$. Moreover

$$
\begin{aligned}
\left(I_{4}\right) & \leq w_{R_{\epsilon}}\left(\left(2 \alpha|I|_{\infty}+2 \alpha|\bar{y}|\right)(1+|\bar{y}|)\right) \\
& \leq w_{R_{\epsilon}}\left(2 \alpha+4 \alpha|\bar{y}|+2 \alpha|\bar{y}|^{2}\right),
\end{aligned}
$$

Now, we use the assumption (H6-0), to have

$$
\begin{aligned}
\left(I_{2}\right) & \leq w\left(|\bar{x}-\bar{y}|(1+|\bar{p}|)+\sigma|\bar{x}-\bar{y}|^{2}\right) \\
& \leq w\left(|\bar{x}-\bar{y}|+\frac{|\bar{x}-\bar{y}|^{4}}{4 \epsilon^{2}}+\sigma|\bar{x}-\bar{y}|^{2}\right) .
\end{aligned}
$$

The estimate of the term $\left(I_{3}\right)$ is prove later.

5. End of the case $\bar{t}>0$. By the above estimate, the viscosity inequality in (23) becomes

$$
\begin{aligned}
\eta & \leq w_{R_{\epsilon}}\left(2 \alpha+4 \alpha|\bar{x}|+2 \alpha|\bar{x}|^{2}\right)+w_{R_{\epsilon}}\left(2 \alpha+4 \alpha|\bar{y}|+2 \alpha|\bar{y}|^{2}\right) \\
& +w\left(|\bar{x}-\bar{y}|+\frac{|\bar{x}-\bar{y}|^{4}}{4 \epsilon^{2}}+\sigma|\bar{x}-\bar{y}|^{2}\right)+\left(I_{3}\right) .
\end{aligned}
$$

From (21) and (22), we have

$$
\begin{gathered}
\lim _{\epsilon, \alpha \rightarrow 0} w_{R_{\epsilon}}\left(2 \alpha+4 \alpha|\bar{x}|+2 \alpha|\bar{x}|^{2}\right)=0 . \\
\lim _{\epsilon, \alpha \rightarrow 0} w_{R_{\epsilon}}\left(2 \alpha+4 \alpha^{2}|\bar{y}|+4 \alpha^{2}|\bar{y}|^{2}\right)=0 . \\
\lim _{\epsilon, \alpha \rightarrow 0} w\left(|\bar{x}-\bar{y}|+\frac{|\bar{x}-\bar{y}|^{4}}{4 \epsilon^{2}}+\sigma|\bar{x}-\bar{y}|^{2}\right)=0 .
\end{gathered}
$$

Now, we send $\alpha$ to zero, the inequality (26) becomes

$$
\begin{aligned}
\eta & \leq \lim _{\alpha \rightarrow 0}\left(w_{R_{\epsilon}}\left(2 \alpha+4 \alpha|\bar{x}|+2 \alpha|\bar{x}|^{2}\right)+w_{R_{\epsilon}}\left(2 \alpha|I|+4 \alpha|\bar{y}|+2 \alpha|\bar{y}|^{2}\right)\right) \\
& +\lim _{\alpha \rightarrow 0} w\left(|\bar{x}-\bar{y}|+\frac{|\bar{x}-\bar{y}|^{4}}{4 \epsilon^{2}}+\sigma|\bar{x}-\bar{y}|^{2}\right)+\lim _{\alpha \rightarrow 0}\left(I_{3}\right) .
\end{aligned}
$$

From now on, we denote by $I_{\epsilon, \alpha}$ the $\operatorname{sum} \frac{1}{2} \sum_{i=k+1}^{N} \frac{\left|\bar{x}_{i}-\bar{y}_{i}\right|^{4}}{4 \epsilon^{4}}$. Then (24) becomes

$$
\overline{\mathcal{E}} \subset\left\{z \in \mathbb{R}^{N-k}:|z|^{2} \geq I_{\epsilon, \alpha}+\sum_{i=k+1}^{N}\left(\bar{x}_{i}^{2}+\bar{y}_{i}^{2}\right)\right\} .
$$

For $\left(I_{3}\right)$ we distinguish two cases :

First case: $\lim _{\alpha \rightarrow 0} I_{\epsilon, \alpha}=0$, in this case we have $\lim _{\alpha \rightarrow 0}\left|\tilde{\pi}_{k}(\bar{p})\right|=0$. Since $\bar{Y}-2 \alpha I$ 
and $\bar{p}-2 \alpha \bar{y}$ remain bounded (independently of $\alpha$ ), we use the assumption (H4'), to obtain

$$
\lim _{\alpha \rightarrow 0}\left(I_{3}\right)=0 .
$$

Now, we send $\epsilon$ to zero in (30) and we use (27), (28) and (29), the inequality (30) becomes $\eta \leq 0$, which is a contradiction since $\eta>0$.

Second case: $\lim _{\alpha \rightarrow 0} I_{\epsilon, \alpha} \neq 0$, in this case, up to extract a subsequence there exists $\delta>0$ such that

$$
I_{\epsilon, \alpha} \geq \delta
$$

The estimate in (24) implies

$$
\overline{\mathcal{E}}=K_{\bar{x}, \bar{t}, u}^{k,+} \backslash K_{\bar{y}, \bar{t}, v}^{k,-} \subset \mathbb{R}^{N-k} \backslash B\left(0, r_{\alpha}\right) .
$$

where $r_{\alpha}=\frac{I_{\epsilon, \alpha}}{\alpha} \geq \frac{\delta}{\alpha}$ tend to $+\infty$ if $\alpha$ tends to zero. Since we know that $\bar{Y}-2 \alpha I$ and $\bar{p}-2 \alpha \bar{y}$ are bounded (independently of $\alpha$ ), then the assumption (H3') implies $\lim _{\alpha \rightarrow 0}\left(I_{3}\right)=0$, and we obtain a contradiction as in the first case.

End of the proof. From above, we have necessarily $\bar{t}=0$, and we conclude as in the precedent section, Step 6 .

\section{Existence Result}

In this Section, we use the classical Perron's method to prove the proof of Corollary 2.1, when the initial front is compact since the one in the non compact case (Corollary 3.1) can be adapted easily. This proof is given by the three following steps.

Step 1. In this step we construct a subsolution $\underline{u} \in \mathcal{C}$ and a supersolution $\bar{u} \in \mathcal{C}$ of our equation (11). We start with the following Lemma.

Lemma 4.1. Let $A \leq-\left(3 L_{1}+2 L_{N-k} L_{1}+2\left(L_{2}+1\right)(2 N+3)\right)$, where $L_{1}, L_{2}$ appear in the assumption (H5) and $C_{N-k}=\mathcal{L}^{N-k}(B(0,1))^{\frac{1}{N-k}}$. Then the function

$$
g(x, t)=\frac{e^{A t}}{1+|x|^{2}}-1
$$

is a smooth subsolution of (11) for $t>0$.

The proof of the Lemma 4.1 is postponed. Now, from the subsolution $g$ for $t>0$, we construct a subsolution in the class $\mathcal{C}$ which satisfy the initial condition. We consider the nondecreasing function $\phi$ from $(-\infty, 0)$ to $\mathbb{R}$ defined by

$$
\phi(t)=\inf _{y \in \mathbb{R}^{N}}\left\{u_{0}(y): g(y, 0) \geq t\right\} .
$$

By the definition of $\phi$ we have

$$
\phi(g(y, 0)) \leq u_{0}(y) .
$$

Starting from this function $\phi$, we will build a regular function which has the property (32). For this reason, we need to prove the following Lemmas. 
Lemma 4.2. The function $\phi_{n}:\left(-\infty, \frac{1}{n}\right) \rightarrow \mathbb{R}$ defined by

$$
\phi_{n}(t)=n \int_{t-\frac{1}{n}}^{t} \phi(s) d s
$$

have the following properties:

i) - $\phi_{n}(t) \leq \phi(t)$ for all $t \in(-\infty, 0)$.

ii)- For all $n$ the function $t \rightarrow \phi_{n}(t)$ is nondecreasing.

iii)- For all $n$ the function $\phi_{n} \in C\left(\left(0, \frac{1}{n}\right)\right)$ and $\phi_{n}(g) \in \mathcal{C}$.

\section{Proof of the Lemma 4.2.}

i)- Since $\phi$ is nondecreasing we have $\phi(s) \leq \phi(t)$ for all $s \leq t$ and

$$
\phi_{n}(t)=n \int_{t-\frac{1}{n}}^{t} \phi(s) d s \leq n \int_{t-\frac{1}{n}}^{t} \phi(t) d s \leq \phi(t)
$$

then $\phi_{n}(t) \leq \phi(t)$ for all $t \in(-\infty, 0)$.

ii)- Let $t^{\prime} \geq t$, by change of the variables $s^{\prime}=s+\left(t^{\prime}-t\right)$, we have

$$
\begin{aligned}
\phi_{n}(t)=n \int_{t-\frac{1}{n}}^{t} \phi(s) d s= & n \int_{t^{\prime}-\frac{1}{n}}^{t^{\prime}} \phi\left(s^{\prime}-\left(t^{\prime}-t\right)\right) d s^{\prime} \\
& \leq n \int_{t^{\prime}-\frac{1}{n}}^{t^{\prime}} \phi\left(s^{\prime}\right) d s^{\prime} \\
& =\phi_{n}\left(t^{\prime}\right)
\end{aligned}
$$

thus $\phi_{n}$ is nondecreasing.

iii)- The function $\phi$ is bounded, then the function $\phi_{n}$ is $C\left(\left(-\infty, \frac{1}{n}\right)\right)$. Since $\phi_{n}$ is nondecreasing by ii) we have

$$
\lim _{|x| \rightarrow \infty} \phi_{n}(g(x, t))=\inf _{x \in \mathbb{R}^{N}} \phi_{n}(g(x, t))=\phi_{n}(-1)=-1
$$

and $\phi_{n}(g(x, t))>-1$ for any $(x, t) \in \mathbb{R}^{N} \times[0, T]$. Then $\phi_{n}(g) \in \mathcal{C}$.

Now we consider the function

$$
\tilde{\phi}_{n}(t)=n \int_{t-\frac{1}{n}}^{t} \phi_{n}(t) d s .
$$

Since $\phi_{n} \in C\left(\left(-\infty, \frac{1}{n}\right)\right)$, then $\tilde{\phi}_{n} \in C^{1}\left(\left(-\infty, \frac{1}{n}\right)\right)$ and the Lemma 4.2 remains valid with the function $\tilde{\phi}_{n}$ (we replace $\phi$ by $\phi_{n}$ ). We argue in the same manner we consider the function

$$
\hat{\phi}_{n}(t)=n \int_{t-\frac{1}{n}}^{t} \tilde{\phi}_{n}(t) d s .
$$

Since $\tilde{\phi}_{n}$ is continuous, then $\hat{\phi}_{n} \in C^{1}\left(-\infty, \frac{1}{n}\right)$ and satisfies the following Lemma. 
Lemma 4.3. The function $\hat{\phi}_{n}$ have the following properties i) - $\hat{\phi}_{n}(t) \leq \phi(t)$ for all $t \in(-\infty, 0)$.

ii)- For all $n$ the function $t \rightarrow \hat{\phi}_{n}(t)$ is nondecreasing.

iii)- For all $n$ the function $\hat{\phi}_{n} \in C^{2}\left(\left(-\infty, \frac{1}{n}\right)\right)$ and $\hat{\phi}_{n}(g) \in \mathcal{C}$.

The proof of this Lemma is similar to the proof of Lemma 4.2,

We use the assumption (H4) which ensures that, the fronts is invariant by nondecreasing changes $u \rightarrow \psi(u)$ (see [10, 8]). Since $g$ is a subsolution of (10) for $t>0$ (Lemma 4.1), and $\hat{\phi}_{n}$ is nondecreasing, the function $\hat{\phi}_{n} \circ g$ is a subsolution of (11) for $t>0$. The definition of $\phi$ and the Lemma 4.2 implies

$$
\hat{\phi}_{n}(g(x, 0)) \leq \phi(g(x, 0)) \leq u_{0}(x) \text { and } \hat{\phi}_{n}(g) \in \mathcal{C},
$$

then $\hat{\phi}_{n} \circ g$ is a subsolution of (1) for all $t \in[0, T]$.

For the construction of the supersolution, we argue in the same manner but, we start in the Lemma 4.1 with $f(x, t)=\frac{e^{A_{0} t}}{1+|x|^{2}}-1$, where $A_{0} \geq$ $\left(3 L_{1}+2 C_{N-k} L_{1}+2(2 N+3)\left(L_{2}+1\right)\right)$, and instead of the function $\phi$, we take the nondecreasing function

$$
\psi(t)=\sup _{y \in \mathbb{R}^{N}}\left\{u_{0}(y): f(y, 0) \leq t\right\} .
$$

Then, there exists a suite of nondecreasing functions $\hat{\psi}_{n} \in C^{1}\left(-\infty, \frac{1}{n}\right)$ such that $\hat{\psi}_{n} \circ f \in \mathcal{C}$ is a supersolution of (1).

Now, it is enough to take $\underline{u}=\hat{\phi}_{n}(g)$ and $\bar{u}=\hat{\psi}_{n}(f)$ to conclude the proof of Step 1 .

Step 2. Consider the set $\mathcal{F}$ of subsolution of (1) $w$ such that $\underline{u} \leq w \leq \bar{u}$. Set then for every $(x, t) \in \mathbb{R}^{N} \times[0, T], v(x, t)=\sup _{w \in \mathcal{F}} w(x, t)$. By Step 1 , the set $\mathcal{F}$ is nonempty and $v$ is well-defined. Thus, we get from the comparison result and classical arguments of the Perron's method that $v$ is a discontinuous solution of (11). For the proof of these classical arguments we refer the reader to Crandall, Ishii, Lions [9] and to Barles [4].

Now, as the subsolutions and the sursolutions do not satisfy the condition initial with equality, we need to using the some arguments used in [1, Proposition 1] and [4, Theorem 4.7] to conclude that $v^{\star}(\cdot, 0)=v_{\star}(\cdot, 0)=u_{0}$. Thus we deduce from the comparison result that $v^{\star}=v_{\star}=v$ which is the desired continuous solution.

Step 3. We show that the solution $v$ built in Step 2 is actually in $\mathcal{C}$. If $w \in \mathcal{F}$, then $w \in \mathcal{C}$ and $w \leq \bar{u}$. Since $\bar{u} \in \mathcal{C}$ we conclude easily $v \in \mathcal{C}$.

Proof of the Lemma 4.1, First, an easy computation show

$$
\left|D^{2} g\right|_{\infty} \leq \frac{2 e^{A t}(2 N+3)}{\left(1+|x|^{2}\right)^{2}}
$$


By (H5), to show that $g$ is subsolution, it suffices to prove be that

$$
\begin{aligned}
(E)= & \frac{\partial g}{\partial t}(x, t)+L_{1}((1+|x|)|D g(x, t)|)\left|\tilde{\pi}_{k}(D g(x, t))\right| \\
& +L_{1}\left(\mathcal{L}^{N-k}\left(K_{x, t, g}^{k,+}\right)^{\frac{1}{N-k}}\left|\tilde{\pi}_{k}(D g(x, t))\right|\right)+\left(L_{2}+1\right)|x|^{2}\left|D^{2} g(x, t)\right|
\end{aligned}
$$

is non positive.

Since $g$ is radial and decreasing in $|x|$, we have

$$
K_{x, t, g}^{k,+} \subset B(0,|x|) \text { and } \mathcal{L}^{N-k}\left(K_{x, t, g}^{k,+}\right) \leq C_{N-k}|x|^{N-k}
$$

or equivalently

$$
\mathcal{L}^{N-k}\left(K_{x, t, g}^{k,+}\right)^{\frac{1}{N-k}} \leq C_{N-k}|x| .
$$

Using (33)), $\left|\tilde{\pi}_{k}(D g(x, t))\right| \leq|D g(x, t)|$, and the above estimate of the upper bound of $\left|D^{2} g\right|_{\infty}$, we have

$$
(E) \leq \frac{A e^{A t}}{1+|x|^{2}}+L_{1}\left((1+|x|) \frac{2 e^{A t}|x|}{\left(1+|x|^{2}\right)^{2}}+\frac{2 e^{A t} C_{N-k}|x|^{2}}{\left(1+|x|^{2}\right)^{2}}\right)+\left(L_{2}+1\right) \frac{2 e^{A t}(2 N+3)}{\left(1+|x|^{2}\right)^{2}}|x|^{2} .
$$

We develop the right-hand side term of (34), we obtain

$$
(E) \leq \frac{e^{A t}\left(A+3 L_{1}+2 C_{N-k} L_{1}+2\left(L_{2}+1\right)(2 N+3)\right)}{1+|x|^{2}} .
$$

Since $A \leq-\left(3 L_{1}+2 C_{N-k} L_{1}+2(2 N+3)\left(L_{2}+1\right)\right)$, then $(E) \leq 0$, and $g$ is a subsolution of (1) for $t>0$.

\section{ApPENDix}

In this Section, we prove a stability result for our nonlocal equation. In fact, we need this result in the proof of Theorem 2.1. Theorem 3.1 and the Perron's method (See Remark 2.1). In [14, this result was formulated in the following way.

Theorem 5.1. If $\left(u_{n}\right)_{n \geq 1}$ is a sequence of upper-semicontinuous viscosity subsolutions of

$$
\frac{\partial u}{\partial t}(x, t)+F_{n}\left(x, t, D u, D^{2} u, K_{x, t, u}^{k,+}\right)=0,
$$

(resp. lower-semicontinuous supersolutions) of

$$
\frac{\partial u}{\partial t}(x, t)+F_{n}\left(x, t, D u, D^{2} u, K_{x, t, u}^{k,-}\right)=0,
$$

where $\left(F_{n}\right)_{n \geq 1}$ is a sequence of uniformly local bounded functions on $\mathbb{R}^{N} \times$ $[0, T] \times \mathbb{R}^{N} \backslash\{0\} \times \mathscr{S}_{N} \times \mathscr{B}_{N-k}$ with satisfying the monotonicity condition (H3). We suppose that, the functions $\left(u_{n}\right)_{n \geq 1}$ are uniformly local bounded 
on $\mathbb{R}^{N} \times[0, T]$, then $\bar{u}=\limsup _{n}^{\star} u_{n}\left(\right.$ resp. $\left.\underline{u}=\liminf _{\star} u_{n}\right)$ is a subsolution (resp. supersolution) of

$$
\frac{\partial u}{\partial t}+\underline{F}\left(x, t, D u, D^{2} u, K_{x, t, u}^{k,+}\right)=0,
$$

respectively of

$$
\frac{\partial u}{\partial t}+\bar{F}\left(x, t, D u, D^{2} u, K_{x, t, u}^{k,-}\right)=0,
$$

where

$$
\underline{F}\left(x, t, p, X, K^{k}\right)=\liminf _{n} F_{n}\left(x_{n}, t_{n}, p_{n}, X_{n}, K_{n}^{k}\right),
$$

respectively

$$
\underline{F}\left(x, t, p, X, K^{k}\right)=\underset{n}{\lim \sup ^{\star}} F_{n}\left(x_{n}, t_{n}, p_{n}, X_{n}, K_{n}^{k}\right),
$$

when $x_{n} \rightarrow x, p_{n} \rightarrow p, X_{n} \rightarrow X, t_{n} \rightarrow t$, and $K_{n}^{k}$ converge to $K^{k}$ if $n$ tends to $\infty$.

Proof of the Theorem 5.1 We give the proof only for $\bar{u}$, that for $\underline{u}$ being similar. Let $\left(x_{0}, t_{0}\right) \in \mathbb{R}^{N} \times[0, T]$ and $\phi \in C^{\infty}\left(\mathbb{R}^{N} \times[0, T]\right)$ such that $\bar{u}-\phi$ has maximum at $\left(x_{0}, t_{0}\right)$. Then from the definition of $\bar{u}$ follows that there is a subsequence of $\left(u_{n}\right)_{n \geq 1}$ that we also denote by $\left(u_{n}\right)_{n \geq 1}$ such that $u_{n}-\phi$ has maximum at $\left(x_{n}, t_{n}\right)$ and

$$
\left(x_{n}, t_{n}\right) \rightarrow\left(x_{0}, t_{0}\right) \quad \text { and } \quad u_{n}\left(x_{n}, t_{n}\right) \rightarrow \bar{u}\left(x_{0}, t_{0}\right) \quad \text { as } \quad \mathrm{n} \rightarrow \infty .
$$

So since $u_{n}$ are subsolutions of (35), we have

$$
\frac{\partial \phi}{\partial t}+F_{n}\left(x_{n}, t_{n}, D \phi\left(x_{n}, t_{n}\right), D^{2} \phi\left(x_{n}, t_{n}\right), K_{x_{n}, t_{n}, u_{n}}^{k,+}\right) \leq 0 .
$$

To continue the proof we need the following Lemma.

Lemma 5.1. Let $u=\lim \sup ^{\star} u_{n}$ where $\left(u_{n}\right)_{n \geq 1}$ is a sequence of uniformly local bounded and upper semicontinuous functions from $\mathbb{R}^{N} \times[0, T]$ to $\mathbb{R}$ and $\left(x_{n}, t_{n}\right)$ a sequence of $\mathbb{R}^{N} \times[0, T]$ such that $\left(x_{n}, t_{n}\right) \rightarrow(x, t)$ and $u_{n}\left(x_{n}, t_{n}\right) \rightarrow u(x, t)$ as $n \rightarrow \infty$, then

$$
\limsup _{n} \mathbb{1}_{K_{x_{n}, t_{n}, u_{n}}^{k,+}}(z) \leq \mathbb{1}_{K_{x, t, u}^{k,+}}(z),
$$

for all $z \in \mathbb{R}^{N}$. Or equivalently we have

$$
\underset{n}{\limsup } K_{x_{n}, t_{n}, u_{n}}^{k,+} \subset K_{x, t, u}^{k,+},
$$

for the set convergence in the topology defined in the introduction.

The proof of the Lemma 5.1 is postponed. Now, we return to the proof of Theorem 5.1. We use the Lemma 5.1 to conclude that

$$
\limsup _{n} K_{x_{n}, t_{n}, u_{n}}^{k,+} \cup K_{x, t, \bar{u}}^{k,+}=K_{x, t, \bar{u}}^{k,+},
$$




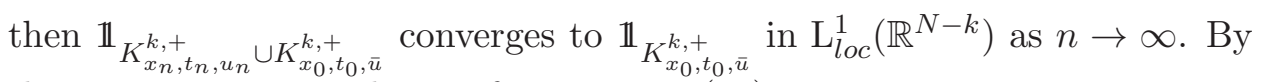
the monotonicity condition of $F_{n}$, we use (37) to write

$$
\frac{\partial \phi}{\partial t}+F_{n}\left(x_{n}, t_{n}, D \phi\left(x_{n}, t_{n}\right), D^{2} \phi\left(x_{n}, t_{n}\right), K_{x_{n}, t_{n}, u_{n}}^{k,+} \cup K_{x_{0}, t_{0}, \bar{u}}^{k,+}\right) \leq 0 .
$$

Moreover, the regularity of the test-function $\phi$ enables us to affirm that

$$
\begin{aligned}
& \frac{\partial \phi}{\partial t}\left(x_{0}, t_{0}\right)+\underline{F}\left(x_{0}, t_{0}, D \phi\left(x_{0}, t_{0}\right), D^{2} \phi\left(x_{0}, t_{0}\right), K_{x_{0}, t_{0}, \bar{u}}^{k,+}\right) \\
& \leq \frac{\partial \phi}{\partial t}\left(x_{0}, t_{0}\right)+\liminf _{n} F_{n}\left(x_{n}, t_{n}, D \phi\left(x_{n}, t_{n}\right), D^{2} \phi\left(x_{n}, t_{n}\right), K_{x_{n}, t_{n}, u_{n}}^{k,+} \cup K_{x_{0}, t_{0}, \bar{u}}^{k,+}\right) \\
& \leq 0 .
\end{aligned}
$$

Then, $\bar{u}$ is a subsolution of( $(35)$.

Proof of Lemma 5.1. For the proof of the Lemma 5.1 we distinguish two cases:

First case: $u(z, t) \geq u(x, t)$, in this case we have $\mathbb{1}_{K_{x, t, u}^{k,+}}(z)=1$ and the result is clear since, obviously

$$
\limsup _{n} \mathbb{1}_{K_{x_{n}, t_{n}, u_{n}}^{k,+}}(z) \leq \mathbb{1}_{K_{x, t, u}^{k,+}}(z)=1 .
$$

Second case: $u(z, t)<u(x, t)$, in this case since $u_{n}\left(x_{n}, t\right) \rightarrow u(x, t)$ and by the definition of $u$ for $n$ large enough we have $u_{n}\left(z, t_{n}\right)<u_{n}\left(x_{n}, t_{n}\right)$ and

$$
\limsup _{n} \mathbb{1}_{K_{x_{n}, t_{n}, u_{n}}^{k,+}}(z) \leq \mathbb{1}_{K_{x, t, u}^{k,+}}(z) .
$$

Remark 5.1. We point out that Lemma 5.1 is not true if we replace ">" by " $\geq$ " in the definition of $K^{k,+}$. It explain why we need to change "testsets" for supersolutions in Definition 1.1 (see Remark 1.1).

\section{Acknowledgments.}

The author would like to thank Guy Barles and Olivier Ley for their great help, their support, their enriching discussions and their many fruitful suggestions in the preparation of this article. I also would like to express my great fullness to Romain Abraham and Maïtine Bergounioux for introducing me to the tomography reconstruction and the studied model. This work was supported by grants of the center region and the national center of scientific research CNRS.

\section{REFERENCES}

[1] Olivier Alvarez and Agnès Tourin. Viscosity solutions of nonlinear integro-differential equations. Ann. Inst. H. Poincaré Anal. Non Linéaire, 13(3):293-317, 1996.

[2] G. Barles, P. Cardaliaguet, O. Ley, and R.Monneau. General results for dislocation type equations. Preprint.

[3] G. Barles, H. M. Soner, and P. E. Souganidis. Front propagation and phase field theory. SIAM J. Control Optim., 31(2):439-469, 1993. 
[4] Guy Barles. Solutions de viscosité des équations de Hamilton-Jacobi, volume 17 of Mathématiques 85 Applications (Berlin) [Mathematics $\& 5$ Applications]. SpringerVerlag, Paris, 1994.

[5] Guy Barles, Samuel Biton, and Olivier Ley. A geometrical approach to the study of unbounded solutions of quasilinear parabolic equations. Arch. Ration. Mech. Anal., 162(4):287-325, 2002.

[6] Guy Barles and Olivier Ley. Nonlocal first-order Hamilton-Jacobi equations modelling dislocations dynamics. Comm. Partial Differential Equations, 31(7-9):11911208, 2006.

[7] Dinten. Bruandet, Peyrin. Binary objects tomographic reconstruction from few noisy $\mathrm{x}$-ray radiographs using a region based curve evolution method. IEEE, 2002.

[8] Yun Gang Chen, Yoshikazu Giga, and Shun'ichi Goto. Uniqueness and existence of viscosity solutions of generalized mean curvature flow equations. J. Differential Geom., 33(3):749-786, 1991.

[9] Michael G. Crandall, Hitoshi Ishii, and Pierre-Louis Lions. User's guide to viscosity solutions of second order partial differential equations. Bull. Amer. Math. Soc. (N.S.), 27(1):1-67, 1992.

[10] L. C. Evans and J. Spruck. Motion of level sets by mean curvature. I [ MR1100206 (92h:35097)]. In Fundamental contributions to the continuum theory of evolving phase interfaces in solids, pages 328-374. Springer, Berlin, 1999.

[11] Y. Giga, S. Goto, H. Ishii, and M.-H. Sato. Comparison principle and convexity preserving properties for singular degenerate parabolic equations on unbounded domains. Indiana Univ. Math. J., 40(2):443-470, 1991.

[12] R. Abraham I. Abraham and M.Bergounioux. An active curve approach for tomographic reconstruction of binary radially symmetric objects. ESAIM: Control, Optimistion and Calculus of Variation.

[13] Stanley Osher and James A. Sethian. Fronts propagating with curvature-dependent speed: algorithms based on Hamilton-Jacobi formulations. J. Comput. Phys., $79(1): 12-49,1988$.

[14] Dejan Slepčev. Approximation schemes for propagation of fronts with nonlocal velocities and Neumann boundary conditions. Nonlinear Anal., 52(1):79-115, 2003.

[15] Panagiotis E. Souganidis. Interface dynamics in phase transitions. In Proceedings of the International Congress of Mathematicians, Vol. 1, 2 (Zürich, 1994), pages 11331144, Basel, 1995. Birkhäuser.

[16] Panagiotis E. Souganidis. Front propagation: theory and applications. In Viscosity solutions and applications (Montecatini Terme, 1995), volume 1660 of Lecture Notes in Math., pages 186-242. Springer, Berlin, 1997.

[17] Mehrdad Soumekh. Binary image reconstruction from four projections. IEEE, 1988.

Laboratoire de Mathématiques et Physique Théorique Université FrançoisRabelais Tours, Fédération Denis Poisson -UMR CNRS 6083, Parc de Grandmont, 37200 Tours. France. Laboratoire de Mathématiques et Applications Physique Mathématique D'OrlÉans, 45067 Orléans Cedex 2

E-mail address: srour@lmpt.univ-tours.fr 